\title{
Der Pyrrhussieg des Egalitarismus. Einkommensungleichheiten in Europa
}

\author{
Die vor acht Jahren in Europa begonnene Finanzmarkt- und Eurokrise hat den sozialen \\ Zusammenhalt in der Europäischen Union (EU) grundlegend erschüttert. Eine mögliche \\ Dimension dieser Erschütterungen ist die Entwicklung der Einkommensungleichheiten. \\ Ergänzend zum Beitrag von Beckfield (in diesem Heft) wird untersucht, wie sich die Un- \\ gleichheit der Einkommen innerhalb und zwischen den EU-27-Ländern - vor allem von \\ 2005 bis 2013 - entwickelt hat. Sowohl der Trend hin zu mehr Ungleichheit innerhalb der \\ Mitgliedstaaten als auch die Angleichung der Einkommensverhältnisse zwischen den \\ Ländern kamen während der Eurokrise zum Stillstand. Weitgehend stabile Einkommens- \\ ungleichheiten gehen allerdings mit einer dramatischen Zunahme der Arbeitslosigkeit in \\ Südeuropa und einem wachsenden Einkommensgefälle zwischen Nord- und Südeuropa \\ einher.
}

MARTIN HEIDENREICH

\section{Einleitung}

Nach einem Sieg gegen die Römer soll König Pyrrhos 279 vor unserer Zeit gesagt haben: „Noch so ein Sieg, und wir sind verloren!" Hieraus leitet sich der Begriff des Pyrrhussieges für einen zu teuer erkauften Erfolg ab. Die Betrachtung der Einkommensungleichheiten in Europa legt dieses Bild nahe. Die von Beckfield (in diesem Heft) vermutete Fortsetzung des langjährigen Trends zu mehr Einkommensungleichheit in der EU wird im Folgenden empirisch überprüft. Während sich Beckfields Untersuchung auf die Entwicklungen der Einkommensungleichheit der EU-15 bezieht und mit dem Jahr 2010 endet, bezieht diese Untersuchung alle EU-27-Länder ein und reicht bis 2013. Anders als von Beckfield angenommen, zeigt sich, dass die Einkommensungleichheiten während der Krise sowohl innerhalb als auch zwischen den 27-EU-Mitgliedstaaten weitgehend stabil geblieben sind.

Die Institutionen des egalitären Kapitalismus, der durch einen ausgebauten Wohlfahrtsstaat, durch Gewerkschaften, ein oftmals hohes Ausbildungsniveau und interregionale Umverteilungen gekennzeichnet ist (Kenworthy 2004), haben verhindert, dass die Einkommen innerhalb der Mitgliedstaaten während der Krise auseinandergedriftet sind. Flankiert wurde diese Stabilität durch einen Wandel vom fürsorgenden zum aktivierenden Wohlfahrtsstaat (Dingeldey 2011) und durch die Einbindung vieler EU-Staaten in eine Währungsunion, in der wirtschaftliche Anpassungen durch monetäre Abwertungen unmöglich sind. Gerade bei einem insgesamt niedrigen Zinsniveau erschwert dies reale Lohn- und Preisanpassungen, was zur Verfestigung des Lohn- und Einkommensgefüges beiträgt. Hierdurch erhöht sich allerdings der Druck auf den Arbeitsmarkt, wenn nicht andere Ausgleichsmechanismen, wie z. B. Staatsverschuldung, internationale Transfers oder Auswanderungen genutzt werden können (Streeck 2013, S. 151). Die Massenarbeitslosigkeit und das sinkende Beschäftigungsniveau insbesondere in Südeuropa verweisen auf die Schattenseiten dieser Entwicklung. Meine These ist somit, dass die Institutionen des egalitären Kapitalismus eine Stabilisierung der Einkommensungleichheit bewirkt haben und hierin auf nationaler Ebene durch Aktivierungspolitiken und auf europäischer Ebene durch die Währungsunion unterstützt wurden. Dieser Sieg über ungleichheitserzeugende technologische und wirtschaftliche Dynamiken könnte sich allerdings als Pyrrhussieg erweisen, da die verfestigten Einkommensrelationen insbesondere in Südeuropa mit einer dramatischen Arbeitsmarktkrise einhergehen.

Im Anschluss an einige konzeptionelle Vorüberlegungen zur Europäisierung sozialer Ungleichheiten (Abschnitt 2) werden im Folgenden die Entwicklung der innerstaatlichen Einkommensungleichheiten (3.1) sowie der objektiven und subjektiven Armut (3.2) nachgezeichnet und die Determinanten der Einkommensungleichheiten untersucht (3.3). Der anschließende Abschnitt behandelt die Entwick- 
lung der europäischen sowie der zwischenstaatlichen Einkommensungleichheiten (4). Abschließend werden die zentralen Ergebnisse resümiert (5).

\section{Die Europäisierung sozialer Ungleichheiten}

Im Zuge zunehmender politischer, wirtschaftlicher und sozialer Verflechtungen in Europa können soziale Ungleichheiten nicht mehr nur im Rahmen von Nationalstaaten analysiert werden. Dies wurde insbesondere in der europäischen Staatsschulden-, Banken- und Wirtschaftskrise seit 2008 deutlich, da die individuelle Lebenssituation zunehmend auch europäischen Entscheidungen zugerechnet wird (Beck 2012). Dies bedroht die Grundlagen der europäischen Integration, wie die zunehmenden zwischenstaatlichen Spannungen zwischen Nord- und Südeuropa, Geber- und Nehmerländern, Euro- und anderen EU-Ländern (nicht zuletzt auch im Zuge der aktuellen Flüchtlingskrise) zeigen. Dies verweist auf eine Europäisierung sozialer Ungleichheiten: Im Zuge der zunehmenden europäischen Verflechtung und politischen Integration der EU entwickeln sich transnationale Standards von Gleichheit und Ungleichheit, durch die unterschiedliche Lebensbedingungen („Heterogenitäten“) zunehmend als Ungleichheiten empfunden und behandelt werden (Heidenreich 2003). Vermutet wird, dass sich Europa gerade in der aktuellen Krise zu einem sozialen Raum entwickelt, der einen erheblichen Einfluss auf die Lebenslagen der Bevölkerung hat und zu einem wichtigen Bezugspunkt für die Wahrnehmung sozialer Ungleichheiten wird. Die Verantwortung für Benachteiligungen und Privilegierungen werden aufgrund der zunehmenden Vertiefung des Integrationsprozesses immer häufiger auch europäischen Entscheidungen zugerechnet - auch wenn der Nationalstaat immer noch die zentrale Bezugseinheit für die Erzeugung, Regulierung und Wahrnehmung sozialer Ungleichheiten ist.

Damit stellt sich die Frage, in welchem Sinne von einer Europäisierung sozialer Ungleichheiten die Rede sein kann. In diesem Beitrag werden zwei verschiedene Bedeutungen unterschieden:

1) Europa als internationaler sozialer Raum: Erstens können innerstaatliche Muster der Entwicklung sozialer Ungleichheiten im Gebiet der EU analysiert und verglichen werden. „Europa“ wird in diesem Fall als sozialer Raum für die Analyse und den Vergleich nationaler Ungleichheiten behandelt.

2) Europa als supranationaler, politisch-administrativ gestalteter Raum: Im Zentrum dieses Verständnisses stehen europäische bzw. zwischenstaatliche Einkommensungleichheiten, deren Entwicklung auch als Ergebnis der europäischen Integration verstanden werden. Politische Entscheidungen und Regeln, die Öffnung der nationalen Kapital-,
Dienstleistungs-, Arbeits- und Gütermärkte, die Einführung einer gemeinsamen Währung oder europaweit koordinierte Austeritätspolitiken können in dieser Perspektive verortet werden (Beckfield 2009).

Diese beiden erläuterten Verständnisse von Europäisierung werden im Folgenden genutzt, um die Frage zu beantworten, wie „Europa“ die Einkommensbedingungen der Bevölkerung von 2005 bis 2013, d. h. vor und während der europäischen Finanzmarkt-, Staatsschulden- und Wirtschaftskrise beeinflusst.

\section{Nationale Einkommens- ungleichheiten in Europa}

Im Folgenden wird zunächst die Entwicklung der Ungleichheiten des verfügbaren Einkommens in den EU-Mitgliedstaaten beschrieben (3.1 und 3.2). Im Anschluss hieran werden diese durch nationale und regionale Kontextfaktoren erklärt (3.3).

\subsection{Die Entwicklung der innerstaatlichen Einkommensungleichheiten}

Vor dem Ausbruch der europäischen Finanz-, Wirtschafts- und Staatsschuldenkrise wurde einerseits eine Annäherung der Lebensstandards zwischen den europäischen Ländern, andererseits eine Zunahme innerstaatlicher Einkommensungleichheiten beobachtet (Beckfield 2009; Heidenreich 2003). Während die erstgenannte Beobachtung durch die regulatorische Vereinheitlichung des europäischen Wirtschaftsraums erklärt werden kann, wird das letztgenannte Ergebnis oftmals als europäische Facette der weltweit zu beobachtenden "großen Kehrtwende“ (Alderson/Nielsen 2002) interpretiert. Im Gegensatz zu dem von Kuznets (1955) prognostizierten Rückgang der Ungleichheiten in entwickelten wohlhabenden Gesellschaften erwartet diese These eine Zunahme der Einkommensungleichheiten aufgrund der Globalisierung der Güter-, Kapital- und Arbeitsmärkte. Andere Autoren verweisen ergänzend auf den technologischen Wandel, infolgedessen immer weniger gering qualifizierte Arbeitskräfte benötigt werden (OECD 2011). Diese Hypothese hat sich für Europa für die Jahrzehnte vor der Krise weitgehend bestätigt (siehe auch den Beitrag von Beckfield in diesem Heft). Ergänzend zu Beckfields Analyse, die mit dem Jahr 2010 endet, wird im Folgenden untersucht, ob und inwieweit sich der Trend zur Vergrößerung der Einkommensungleichheiten bis 2013 fortgesetzt hat. Neben den von Beckfield betrachteten EU-15-Ländern werden hier zusätzlich die EU-27-Länder (also noch ohne Kroatien) sowie die Länder der Eurozone (einmal mit und einmal ohne Lettland) untersucht. 
TABELLE 1

Gini-Koeffizient* des verfügbaren Äquivalenzeinkommens, 1999, 2005-2013

\begin{tabular}{|c|c|c|c|c|c|c|c|c|c|c|c|c|}
\hline Erhebungsjahr & 1999 & 2005 & 2006 & 2007 & 2008 & 2009 & 2010 & 2011 & 2012 & 2013 & $1999-2008$ & $2008-2013$ \\
\hline EU-27 Länder & & 30,6 & 30,3 & 30,6 & 30,9 & 30,5 & 30,4 & 30,7 & 30,4 & 30,5 & & $-0,4$ \\
\hline EU-15 Länder & 29 & 30,0 & 29,6 & 30,3 & 30,8 & 30,5 & 30,5 & 30,8 & 30,4 & 30,4 & 1,8 & $-0,4$ \\
\hline Euroraum (18 Länder) & & 29,3 & 29,3 & 30,0 & 30,4 & 30,2 & 30,2 & 30,5 & 30,3 & 30,6 & & 0,2 \\
\hline Euroraum (17 Länder) & & 29,3 & 29,2 & 30,0 & 30,3 & 30,2 & 30,2 & 30,5 & 30,3 & 30,6 & & 0,3 \\
\hline Großbritannien & 32 & 34,6 & 32,5 & 32,6 & 33,9 & 32,4 & 32,9 & 33,0 & 31,3 & 30,2 & 1,9 & $-3,7$ \\
\hline Niederlande & 26 & 26,9 & 26,4 & 27,6 & 27,6 & 27,2 & 25,5 & 25,8 & 25,4 & 25,1 & 1,6 & $-2,5$ \\
\hline Lettland & $34^{1}$ & 36,2 & 38,9 & 35,4 & 37,5 & 37,5 & 35,9 & 35,1 & 35,7 & 35,2 & 3,5 & $-2,3$ \\
\hline Rumänien & $29^{1}$ & 31 & 33 & 37,8 & 36,0 & 34,9 & 33,3 & 33,2 & 33,2 & 34,0 & 7 & $-2,0$ \\
\hline Belgien & 29 & 28,0 & 27,8 & 26,3 & 27,5 & 26,4 & 26,6 & 26,3 & 26,5 & 25,9 & $-1,5$ & $-1,6$ \\
\hline Portugal & 36 & 38,1 & 37,7 & 36,8 & 35,8 & 35,4 & 33,7 & 34,2 & 34,5 & 34,2 & $-0,2$ & $-1,6$ \\
\hline Polen & $30^{1}$ & 35,6 & 33,3 & 32,2 & 32,0 & 31,4 & 31,1 & 31,1 & 30,9 & 30,7 & 2 & $-1,3$ \\
\hline Finnland & 24 & 26,0 & 25,9 & 26,2 & 26,3 & 25,9 & 25,4 & 25,8 & 25,9 & 25,4 & 2,3 & $-0,9$ \\
\hline Österreich & 26 & 26,3 & 25,3 & 26,2 & 27,7 & 27,5 & 28,3 & 27,4 & 27,6 & 27,0 & 1,7 & $-0,7$ \\
\hline Bulgarien & $25^{1}$ & 25 & 31,2 & 35,3 & 35,9 & 33,4 & 33,2 & 35,0 & 33,6 & 35,4 & 10,9 & $-0,5$ \\
\hline Deutschland & 25 & 26,1 & 26,8 & 30,4 & 30,2 & 29,1 & 29,3 & 29,0 & 28,3 & 29,7 & 5,2 & $-0,5$ \\
\hline Malta & $30^{1}$ & 27,0 & 27,1 & 26,3 & 28,1 & 27,4 & 28,6 & 27,2 & 27,1 & 27,9 & $-1,9$ & $-0,2$ \\
\hline Tschechien & & 26,0 & 25,3 & 25,3 & 24,7 & 25,1 & 24,9 & 25,2 & 24,9 & 24,6 & & $-0,1$ \\
\hline Irland & 32 & 31,9 & 31,9 & 31,3 & 29,9 & 28,8 & 30,7 & 29,8 & 29,9 & 30,0 & $-2,1$ & 0,1 \\
\hline Litauen & 311 & 36,3 & 35,0 & 33,8 & 34,5 & 35,9 & 37,0 & 33,0 & 32,0 & 34,6 & 3,5 & 0,1 \\
\hline Frankreich & 29 & 27,7 & 27,3 & 26,6 & 29,8 & 29,9 & 29,8 & 30,8 & 30,5 & 30,1 & 0,8 & 0,3 \\
\hline Slowakei & & 26,2 & 28,1 & 24,5 & 23,7 & 24,8 & 25,9 & 25,7 & 25,3 & 24,2 & & 0,5 \\
\hline Schweden & 22 & 23,4 & 24,0 & 23,4 & 24,0 & 24,8 & 24,1 & 24,4 & 24,8 & 24,9 & 2 & 0,9 \\
\hline Griechenland & 34 & 33,2 & 34,3 & 34,3 & 33,4 & 33,1 & 32,9 & 33,5 & 34,3 & 34,4 & $-0,6$ & 1,0 \\
\hline Slowenien & $22^{1}$ & 23,8 & 23,7 & 23,2 & 23,4 & 22,7 & 23,8 & 23,8 & 23,7 & 24,4 & 1,4 & 1,0 \\
\hline Italien & 30 & 32,8 & 32,1 & 32,2 & 31,0 & 31,5 & 31,2 & 31,9 & 31,9 & 32,5 & 1 & 1,5 \\
\hline Spanien & 33 & 32,2 & 31,9 & 31,9 & 31,9 & 32,9 & 33,5 & 34,0 & 34,2 & 33,7 & $-1,1$ & 1,8 \\
\hline Estland & $36^{1}$ & 34,1 & 33,1 & 33,4 & 30,9 & 31,4 & 31,3 & 31,9 & 32,5 & 32,9 & $-5,1$ & 2,0 \\
\hline Dänemark & 21 & 23,9 & 23,7 & 25,2 & 25,1 & 26,9 & 26,9 & 27,8 & 28,1 & 27,5 & 4,1 & 2,4 \\
\hline Luxemburg & 27 & 26,5 & 27,8 & 27,4 & 27,7 & 29,2 & 27,9 & 27,2 & 28,0 & 30,4 & 0,7 & 2,7 \\
\hline Ungarn & $26^{1}$ & 27,6 & 33,3 & 25,6 & 25,2 & 24,7 & 24,1 & 26,8 & 26,9 & 28,0 & $-0,8$ & 2,8 \\
\hline Kroatien & & 30 & 28 & 29 & 28 & 27 & 31,6 & 31,2 & 30,9 & 30,9 & & 2,9 \\
\hline Zypern & & 28,7 & 28,8 & 29,8 & 29,0 & 29,5 & 30,1 & 29,2 & 31,0 & 32,4 & & 3,4 \\
\hline
\end{tabular}

*Eurostat berechnet die Gini-Indizes für die EU und die Eurozone als mit der Bevölkerungszahl gewichtetes Mittel der nationalen Werte. Die Gini-Koeffizienten sind skaliert von 0 bis 100. Die letzte Spalte - die auch als Sortierkriterium dient - wurde berechnet als Differenz der Gini-Indizes für 2008 und 2013.

1 Die Zahl bezieht sich auf das Jahr 2000.

Quelle: Eurostat: EU-SILC, Tabelle (ilc_di12).

Die Analyse stützt sich vor allem auf eigene Auswertungen der Haushaltsbefragung „Leben in Europa“ (EU-SILC) ${ }^{1}$ und auf weitere, insbesondere von Eurostat bereitgestellte Daten. Als Einkommen wird im Folgenden das verfügbare, kaufkraftbereinigte, bedarfsgewichtete Haushaltsnettoäquivalenzeinkommen (ohne Steuern plus Sozialleistungen) verstanden. Als Maß für die Einkommensungleichheiten wird der Gini-Index herangezogen.

In den Debatten um die sozialen Folgen der Krise wird unterstellt, dass die nationalen Einkommensungleichheiten in der EU und die Kluft zwischen Insidern und Outsidern zugenommen haben (Blyth 2013, S. 15; siehe auch den Beitrag von Beckfield in diesem Heft). Den hier verwendeten Daten zufolge sind die durchschnittlichen, am Gini-Index gemessenen, nationalen Einkommensungleichheiten in der EU-27, in der EU-15 und in der Eurozone von 2008 bis 2013 im

1 Anzumerken ist, dass sich die Angaben zu den verfügbaren Einkommen bei EU-SILC immer auf das vorangehende Jahr beziehen. Dies gilt auch für die Angaben von Eurostat; daher wird im Folgenden immer das Erhebungsjahr, nicht das Jahr, in dem das Einkommen erzielt wurde, angegeben. Die für das Erhebungsjahr 2013 ausgewiesenen Werte beziehen sich somit auf die 2012 erzielten Einkommen. Die Stärken und Schwächen dieser Daten und die verwendeten Methoden werden ausführlicher in Heidenreich (2010) diskutiert. 
TABELLE 2

Entwicklung der Einkommensungleichheiten in der EU-15 und EU-25**, Erhebungsjahre 2005-2013

\begin{tabular}{lllllll}
\hline Erhebungsjahr & \multicolumn{5}{c}{ EU-15 } \\
\hline & Gini & D9/D1 & MLD & Theil & A(2) \\
\hline 2005* & 0,292 & 3,8113 & 0,150 & 0,144 & 0,290 \\
\hline $2006^{*}$ & 0,288 & 3,809 & 0,148 & 0,138 & 0,296 \\
\hline 2007 & 0,296 & 3,946 & 0,155 & 0,147 & 0,298 \\
\hline 2008 & 0,298 & 3,943 & 0,157 & 0,150 & 0,320 \\
\hline 2009 & 0,294 & 3,901 & 0,152 & 0,144 & 0,303 \\
\hline 2010 & 0,294 & 3,893 & 0,154 & 0,145 & 0,316 \\
\hline 2011 & 0,297 & 3,953 & 0,160 & 0,148 & 0,413 \\
\hline 2012 & 0,298 & 4,012 & 0,164 & 0,148 & 0,528 \\
\hline 2013 & 0,300 & 4,034 & 0,165 & 0,151 & 0,359 \\
\hline
\end{tabular}

\begin{tabular}{c|c|c|c}
\hline \multicolumn{4}{c}{ EU-25 } \\
\hline Gini & D9/D1 & MLD & Theil \\
\hline 0,333 & 5,435 & 0,210 & 0,209 \\
\hline 0,326 & 5,251 & 0,198 & 0,176 \\
\hline 0,331 & 5,147 & 0,199 & 0,182 \\
\hline 0,327 & 4,905 & 0,193 & 0,179 \\
\hline 0,321 & 4,678 & 0,183 & 0,171 \\
\hline 0,319 & 4,657 & 0,183 & 0,199 \\
\hline 0,319 & 4,602 & 0,184 & 0,169 \\
\hline 0,318 & 4,593 & 0,185 & 0,167 \\
\hline 0,318 & 4,590 & 0,184 & 0,168 \\
\hline
\end{tabular}

*Für 2005 und 2006 sind die Daten für Malta nicht verfügbar.

**Die Daten für Rumänien und Bulgarien sind erst ab 2007 und die für Kroatien erst ab 2011 verfügbar. Daher werden hier die

EU-25 anstelle der EU-27 oder EU-28 ausgewiesen.

Anmerkung: Zur Erläuterung der Abkürzungen vgl. Fußnote 2.

Quelle: Berechnungen des Autors auf Grundlage von EU-SILC (200-52013).

Wesentlichen stabil (Tabelle 1). In einigen Ländern mit einem deutlichen Rückgang des durchschnittlich verfügbaren Einkommens, wie Großbritannien oder Lettland (siehe hierzu Tabelle 3), nahmen die Einkommensungleichheiten sogar ab. In acht EU-Staaten (Zypern, Kroatien, Ungarn, Luxemburg, Dänemark, Estland, Spanien und Italien) erhöhten sich die Ungleichheiten um mehr als einen Prozentpunkt, während sie in sieben Ländern geringer wurden. In zwei der fünf Programmländer (Zypern und Spanien) sind die Einkommensungleichheiten größer geworden, während sie in Portugal zurückgegangen und in Griechenland sowie Irland in etwa gleich geblieben sind. Die relative Stabilität nationaler Ungleichheitsmuster ist ein deutlicher Unterschied zu den ersten zehn Jahren nach der Einführung des Euro (1999-2008), in der der Gini-Koeffizient in 13 EU-Ländern um mehr als einen Prozentpunkt anstieg, während er nur in fünf Ländern deutlich zurückging. Der Trend zu steigenden Einkommensungleichheiten wurde somit in der Krise gestoppt.

Mit den verfügbaren Daten können auch weitere Ungleichheitsmaße berechnet werden, die jeweils einen Bereich der Einkommensverteilung besonders hervorheben (Tabelle 2). ${ }^{2}$ Festgehalten werden kann, dass in der EU-25 bei nahezu allen Indikatoren abnehmende Ungleichheiten auch in der Eurokrise zu verzeichnen sind. (Eine Ausnahme hiervon ist der besonders „ungleichheitssensible“ Atkinson(2)Koeffizient). Allerdings nehmen die Einkommensungleichheiten in den EU-Mitgliedstaaten, die der EU schon vor den Erweiterungsrunden von 2004 - 2013 angehörten (EU15) - ab $2010 \mathrm{zu}$.

\subsection{Objektive und subjektive Armut}

Die Stabilität bei der innerstaatlichen Einkommensungleichheit gilt auch für die Quote der monetären Armut, d. h. den Anteil der Bevölkerung, der über weniger als $60 \%$ des durchschnittlichen nationalen Einkommens verfügt (Tabelle 3). Diese Quote lag in der EU 2008 bei 16,4 und 2013 bei 16,5\%. In den alten Mitgliedstaaten ist die Armut von 16,2 (2008) auf 16,3\% (2013) angestiegen. Etwas deutlicher war der für die Eurozone (EU-18) beobachtete Anstieg von 15,9\% (2008) auf 16,5\% (2013) (ohne Tabelle).

Diese weitgehende Stabilität bedeutet keinesfalls, dass die Krise keinen Einfluss auf soziale Ungleichheiten hat. Im Gegenteil: Im Juni 2014 waren zwei Drittel aller Europäer nicht mit der Art und Weise zufrieden, wie mit den Fragen von sozialer Ungleichheit und Armut umgegangen wird (Eu-
2 Jedes Ungleichheitsmaß stützt sich auf normative Verteilungsannahmen. Der Gini-Index geht davon aus, dass jede Abweichung von einer Gleichverteilung wichtig ist und reagiert daher besonders empfindlich auf Abweichungen vom Mittelwert. Das Dezilverhältnis D9/D1 gibt Auskunft über das Verhältnis des obersten zum untersten Zehntels und somit über die gesamte Spannweite der Einkommensverteilung. DerTheil-Index reagiert besonders empfindlich auf Einkommensunterschiede im oberen Bereich der Verteilung, während die mittlere logarithmische Abweichung
(MLD) besonders empfindlich für Veränderungen im unteren Einkommensbereich ist. Der Atkinson-Index $A(\varepsilon)$ berücksichtigt gezielt verschiedene Grade der Aversion gegen Ungleichheit. Der hier ausgewiesene Index A(2) impliziert, dass Einkommensumverteilungen am unteren Ende der Einkommensverteilung einen erheblich höheren Nutzen als Transfers am oberen Ende haben. Der Anteil der Armen ( $60 \%$ und weniger des mittleren Einkommens) ignoriert die Mitte der Verteilung und die Unterschiede zwischen mehr oder minder gut ausgestatteten Armen. 
Einkommen und Armut im nationalen und europäischen Kontext, 2008 und 2013

Angaben in Prozent und Euro

\begin{tabular}{|c|c|c|c|c|c|c|c|c|c|}
\hline & $\begin{array}{c}\text { Armut } \\
\text { (nationale } \\
\text { Schwelle, } \\
2008 \text { )* } \\
\text { in \% } \\
\end{array}$ & $\begin{array}{c}\text { Armut } \\
\text { (nationale } \\
\text { Schwelle, } \\
\text { 2013)* } \\
\text { in \% }\end{array}$ & $\begin{array}{l}\text { Armut } \\
\text { (europ. } \\
\text { Schwelle, } \\
\text { 2008)* } \\
\text { in \% }\end{array}$ & $\begin{array}{c}\text { Armut } \\
\text { (europ. } \\
\text { Schwelle, } \\
\text { 2013)* } \\
\text { in \% }\end{array}$ & $\begin{array}{c}\text { Subjektive } \\
\text { Armut } \\
(2008)^{* *} \\
\text { in } \%\end{array}$ & $\begin{array}{c}\text { Subjektive } \\
\text { Armut }(2013)^{* *} \\
\text { in } \%\end{array}$ & $\begin{array}{c}\text { Verfügbares } \\
\text { Einkommen } \\
\text { (Median, } \\
\text { KKS, 2008) } \\
\text { in Euro } \\
\end{array}$ & $\begin{array}{c}\text { Verfügbares } \\
\text { Einkommen } \\
\text { (Median, } \\
\text { KKS, 2013) } \\
\text { in Euro } \\
\end{array}$ & $\begin{array}{c}\text { Einkommens- } \\
\text { entwicklung } \\
(2008-13) \\
\text { in } \%\end{array}$ \\
\hline Belgien & 14,7 & 15 & 9,1 & 6,6 & 21,6 & 20,9 & 16.751 & 19.579 & 16,9 \\
\hline Bulgarien & 21,4 & 21 & 85,4 & 77,2 & 63,6 & 65,2 & 4.783 & 5.901 & 23,4 \\
\hline Dänemark & 11,5 & 11,7 & 5,6 & 5,5 & 8 & 12,3 & 17.596 & 19.321 & 9,8 \\
\hline Deutschland & 14,9 & 15,9 & 8,4 & 7,6 & 6,8 & 9,1 & 18.002 & 19.441 & 8,0 \\
\hline Estland & 19,4 & 18,5 & 64,1 & 54,8 & 11,1 & 23,3 & 7.556 & 8.613 & 14,0 \\
\hline Finnland & 13,6 & 11,8 & 8,4 & 4,7 & 8,1 & 6,8 & 16.556 & 19.178 & 15,8 \\
\hline Frankreich & 12,5 & 13,7 & 5,8 & 5,4 & 15,9 & 20,6 & 17.493 & 19.216 & 9,8 \\
\hline Griechenland & 19,8 & 22,7 & 28,9 & 50,7 & 54,5 & 78,3 & 12.032 & 9.052 & $-24,8$ \\
\hline Irland & 15,4 & 14,1 & 7,4 & 11,6 & 23,6 & 36,8 & 18.169 & 15.968 & $-12,1$ \\
\hline Italien & 18,5 & 19 & 17,0 & 18,8 & 38,6 & 41,6 & 15.262 & 15.223 & $-0,3$ \\
\hline Kroatien & & 19,5 & & 65,7 & & 62,5 & & 7.414 & \\
\hline Lettland & 25,8 & 19,3 & 61,7 & 48,2 & 39,7 & 54,4 & 7.119 & 6.443 & $-9,5$ \\
\hline Litauen & 20 & 20,5 & 67,0 & 64,6 & 25,3 & 32,9 & 6.850 & 7.282 & 6,3 \\
\hline Luxemburg & 13,3 & 15,7 & 1,3 & 1,7 & 7,2 & 13,3 & 26.943 & 28.030 & 4,0 \\
\hline Malta & 15,2 & 15,7 & 21,2 & 15,3 & 37,2 & 36,5 & 13.264 & 15.056 & 13,5 \\
\hline Niederlande & 10,2 & 10,2 & 3,4 & 4,3 & 10,9 & 15,4 & 19.142 & 19.221 & 0,4 \\
\hline Österreich & 15,2 & 14,4 & 6,8 & 6 & 13,9 & 14 & 18.932 & 20.904 & 10,4 \\
\hline Polen & 16,8 & 17,3 & 69,5 & 50,8 & 35,9 & 32,9 & 6.731 & 9.175 & 36,3 \\
\hline Portugal & 18,5 & 18,7 & 44,4 & 47,3 & 46,7 & 46,9 & 9.514 & 9.820 & 3,2 \\
\hline Rumänien & 23,4 & 22,4 & 95,7 & 94,9 & 48,2 & 50,8 & 3.068 & 3.944 & 28,6 \\
\hline Schweden & 12,1 & 14,6 & 6,2 & 5,5 & 8,6 & 6,5 & 17.799 & 20.516 & 15,3 \\
\hline Slowakei & 10,8 & 12,8 & 75,9 & 45,4 & 34,9 & 36,5 & 6.761 & 9.572 & 41,6 \\
\hline Slowenien & 12,3 & 14,5 & 14,5 & 17,2 & 26,1 & 33,1 & 13.812 & 14.212 & 2,9 \\
\hline Spanien & 20,4 & 20,2 & 23,4 & 23,2 & 31,8 & 38,8 & 13.598 & 14.250 & 4,8 \\
\hline Tschechien & 9 & 8,6 & 40,0 & 33,9 & 28,1 & 31,8 & 9.725 & 10.802 & 11,1 \\
\hline Ungarn & 12,3 & 14,3 & 77,3 & 68,1 & 44 & 53,9 & 6.597 & 7.404 & 12,2 \\
\hline $\begin{array}{l}\text { Vereinigtes } \\
\text { Königreich }\end{array}$ & 18,5 & 15,9 & 9,3 & 12,4 & 17,2 & 21,1 & 18.389 & 16.826 & $-8,5$ \\
\hline Zypern & 15,9 & 15,3 & 6,6 & 8,4 & 51,7 & 59,4 & 18.241 & 17.165 & $-5,9$ \\
\hline EU-28 & 16,4 & 16,5 & 24,9 & 23,6 & 25,0 & 28,8 & 14.577 & 15.304 & 5,0 \\
\hline
\end{tabular}

*Die Armutsquoten (in Prozent der nationalen Bevölkerung) wurden mit Bezug auf 60 \% des nationalen bzw. europäischen Medianeinkommens berechnet.

**Als subjektive Armut wird der Anteil der Personen gezählt, die die finanzielle Situation ihres Haushalts als schlecht oder sehr schlecht beurteilen. Die Entwicklung des verfügbaren, kaufkraftbereinigten Medianeinkommens wurde der Eurostat-Tabelle ilc_di04 entnommen. Für 2008 fehlen in EU-SILC die Daten für Kroatien.

Quelle: Berechnungen des Autors auf Grundlage von EU-SILC.

ropäische Kommission 2014a). Für ein Drittel sind soziale Ungleichheiten eine der beiden größten Herausforderungen in Europa (Europäische Kommission 2014b). Ebenfalls ein Drittel fühlte sich im Frühjahr 2014 von Armut bedroht - ein Anteil, der insbesondere in Spanien, Griechenland, Kroatien und Zypern sehr hoch war und stark zugenommen hat (Europäische Kommission 2014c). Die sozialen Ungleichheiten in Europa bedrohen somit den sozialen Zusammenhalt in Europa sowie den europäischen Integrationsprozess, ohne dass sich dies in den gewählten, auf nationaler Ebene ansetzenden Ungleichheitsindikatoren niederschlägt.
Eine Ursache für diesen Widerspruch könnte sein, dass der nationale Bezugsrahmen, der den Gini-Indizes zugrunde liegt, die Wahrnehmung sozialer Ungleichheiten nicht angemessen widerspiegelt. Neben dem nationalen Bezugsrahmen sind auch transnationale, insbesondere europäische Vergleichsmaßstäbe wichtig. Die Einschätzung der finanziellen Situation des eigenen Haushalts ist eng mit dem kaufkraftbereinigten Haushaltseinkommen korreliert (Tabelle 3): $r^{2}$ liegt hier bei 0,53. Am Beispiel von Griechenland kann diese Diskrepanz zwischen nationalen Ungleichheits- und Armutsindikatoren auf der einen Seite sowie subjek- 
tiver Armut auf der anderen Seite veranschaulicht werden. Während die nationale Armutsquote in der Krise um etwa drei Prozentpunkte (von 19,8 auf 22,7\%) anstieg, schrumpfte das verfügbare Einkommen kaufkraftbereinigt zwischen 2008 und 2013 um 24,8\%. Letzteres erklärt sicherlich eher, warum der Anteil der Personen, die über finanzielle Schwierigkeiten berichteten, von 54,5 auf 78,3\% anstieg. Nationale Ungleichheitsmaße, wie die genannte Armutsquote oder der Gini-Index, erfassen somit nicht den Basiseffekt, d. h. die Verringerung oder Erhöhung des nationalen Einkommensniveaus. Der Rückgang des verfügbaren Einkommens kann somit die Zunahme des wahrgenommenen finanziellen Stresses plausibler erklären als der leichte Anstieg der nationalen Armuts- und Ungleichheitsmaße. Wichtig sind somit die wirtschaftliche Entwicklung des Landes insgesamt und insbesondere die Höhe des Einkommens, über das ein Haushalt verfügen kann. Bei diesem Indikator zeigen sich sehr unterschiedliche Entwicklungen in Europa. Dieses Einkommen hat sich (in gerundeten Werten) in der Slowakei zwischen 2008 und 2013 um 42 \%, in Polen um 36\%, in Rumänien um $29 \%$ und in Bulgarien um $23 \%$ erhöht, während es in Griechenland um ein Viertel, in Irland um 12\% und in Lettland und Großbritannien um 10 bzw. $9 \%$ gesunken ist (Tabelle 3).

Gleichzeitig aber ist ein anderer Effekt zu beobachten die Europäisierung und Transnationalisierung des Bezugsrahmens für subjektive Armuts- und Deprivationserfahrungen (Fahey 2007; Heidenreich/Härpfer 2010). Whelan und Maître (2009, S. 118) beschreiben dies wie folgt: „People perceive themselves as part of a larger European stratification system. Furthermore, the perception of being advantaged or disadvantaged within this system would have to play an important role in individuals' evaluations of their own life circumstances." Der deutliche Anstieg der subjektiv wahrgenommenen Armut nicht nur in Ländern, in denen das Einkommen zurückgegangen ist (Griechenland, Irland), sondern auch in ärmeren EU-Ländern mit einer positiven Einkommensentwicklung (insbesondere in Estland, Ungarn, Zypern, Litauen, Slowenien und Spanien), deutet auf eine solche Europäisierung und Transnationalisierung der Referenzpunkte für die Bewertung der eigenen finanziellen Situation hin. In Estland ist beispielsweise die Armutsquote zurückgegangen und das Einkommen hat sich deutlich erhöht. Gleichzeitig hat sich der Anteil der Haushalte, die kaum mit ihrem Einkommen auskommen, mehr als verdoppelt. Auch die Konsumgewohnheiten, die Anspruchsniveaus und die wahrgenommenen Ungleichheiten haben sich an die anderen EU-Staaten angenähert. Europa ist somit auch in subjektiver Hinsicht ein sozialer Raum, der auch für die Wahrnehmung der sozialen Ungleichheiten zunehmend wichtiger wird.

\subsection{Determinanten der innerstaatlichen und regionalen Einkommensungleichheiten}

Im Folgenden soll die Frage geklärt werden, warum die objektiven innerstaatlichen Einkommensungleichheiten in der Krise weitgehend stabil geblieben sind. Hierbei hilft ein
Blick auf die wirtschaftsstrukturellen und institutionellen Bestimmungsfaktoren der in Tabelle 1 beschriebenen Einkommensungleichheiten. Sofern dies auf der Grundlage von EU-SILC-Daten möglich ist, werden hierbei auch die regionalen Einkommensungleichheiten berücksichtigt, da sich diese auch innerhalb eines Landes deutlich unterscheiden können. So hat etwa der Großraum London einen um fast fünf Punkte höheren Gini-Index als Wales. Diese nationalen und regionalen Muster von Einkommensungleichheiten sollen durch regionale und nationale Kontextfaktoren sowie durch supranationale Prozesse erklärt werden (Heidenreich 2010). Zunächst soll überprüft werden, ob die klassischen egalitären Institutionen (sozialstaatliche Absicherungen, Gewerkschaften, Bildungssysteme) einen Einfluss auf das Ungleichheitsniveau haben. Im Zentrum egalitaristischer Gesellschaftsmodelle steht das industrielle Normalarbeitsverhältnis. Als Indikator hierfür wird der Anteil der Industriebeschäftigten einbezogen. Weiterhin soll überprüft werden, ob Aktivierungspolitiken einen Beitrag zur Verringerung von Einkommensungleichheiten leisten, indem sie die Zahl der Beschäftigten pro Haushalt erhöhen. Als Indikator hierfür wird der Anteil der weiblichen Erwerbstätigen genommen. Vermutet werden kann auch, dass die Globalisierung der Wirtschaft - gemessen an der Integration der Waren-, Dienstleistungs-, Kapital- und Arbeitsmärkte - und die Politik der Haushaltskonsolidierung („Austerität“) zu einer höheren Ungleichheit in der EU beitragen (Blyth 2013).

Die entsprechenden Hypothesen werden für den Zeitraum von 2005 bis 2013 (Spalte 1), für die Jahre vor (2005-2008, Spalte 2) und für die Jahre während der Krise (2009-2013, Spalte 3) getestet (Tabelle 4). Es zeigt sich, dass ein höherer Anteil von Industriebeschäftigten und ausgebaute wohlfahrtsstaatliche Absicherungen in allen drei Zeiträumen mit geringeren Einkommensungleichheiten einhergingen. Einkommen in der Industrie sind egalitärer verteilt als im Dienstleistungssektor. Eine höhere Frauenerwerbstätigenquote ging insbesondere in der Krise mit einer egalitäreren Einkommensstruktur einher, da eine stärkere Erwerbsbeteiligung von Frauen das Einkommen und die Anzahl der Erwerbspersonen pro Haushalt auch in weniger wohlhabenden Haushalten erhöhen. Ein höherer Anteil qualifizierter Arbeitnehmer und stärkere Gewerkschaften verringern die Einkommensungleichheiten gerade auch in der Krise. Die klassischen egalitären Institutionen der Industriegesellschaft werden ergänzt durch Aktivierungspolitiken, die durch eine Erhöhung der Beschäftigungszahlen auch zu egalitäreren Haushaltseinkommen beitragen. Die wirtschaftliche Integration in die europäische und globale Wirtschaft hat in der Krise einen signifikant negativen Einfluss auf die Einkommensungleichheit. Sparmaßnahmen bzw. Austeritätspolitiken haben während der Krise keinen signifikanten Effekt.

Festgehalten werden kann: Anders als von Beckfield vermutet, sind die nationalen Einkommensungleichheiten in der EU in der Krise nicht mehr angestiegen. Nach wie vor werden die Muster der nationalen und regionalen 
Bestimmungsfaktoren der nationalen und regionalen Einkommensungleichheiten vor und während der Großen Rezession (2005-2013) in 27 EU-Mitgliedstaaten

\begin{tabular}{|c|c|c|c|}
\hline & $2005-2013$ & $2005-2008$ & $2009-2013$ \\
\hline $\begin{array}{l}\text { Anteil der Beschäftigten in der Industrie } \\
\text { (in \% aller Erwerbstätigen) }\end{array}$ & $\begin{array}{l}-0,09+ \\
(-1,82)\end{array}$ & $\begin{array}{l}-0,13^{*} \\
(-2,25)\end{array}$ & $\begin{array}{l}-0,15^{* *} \\
(-6,70)\end{array}$ \\
\hline $\begin{array}{l}\text { Erwerbstätigenquote von Frauen } \\
\text { (in \% der 15-64-jährigen Frauen) }\end{array}$ & $\begin{array}{l}-0,05 \\
(-1,17)\end{array}$ & $\begin{array}{c}-0,06 \\
(-1,37)\end{array}$ & $\begin{array}{l}-0,08+ \\
(-1,95)\end{array}$ \\
\hline $\begin{array}{l}\text { Mittleres Ausbildungsniveau } \\
\text { (in \% der 15-64-jährigen Einwohner) }\end{array}$ & $\begin{array}{c}-0,03 \\
(-1,33)\end{array}$ & $\begin{array}{c}-0,06 \\
(-1,53)\end{array}$ & $\begin{array}{l}-0,09 * * \\
(-3,87)\end{array}$ \\
\hline $\begin{array}{l}\text { Sozialausgaben } \\
\text { (in \% des Bruttoinlandproduktes) }\end{array}$ & $\begin{array}{c}-0,08 \\
(-1,13)\end{array}$ & $\begin{array}{l}-0,31 * * \\
(-4,20)\end{array}$ & $\begin{array}{l}-0,26 * * \\
(-4,08)\end{array}$ \\
\hline $\begin{array}{l}\text { Gewerkschaftlicher Organisationsgrad } \\
\text { (in \% der Arbeitnehmer) }\end{array}$ & $\begin{array}{l}-0,05 \\
(-1,52)\end{array}$ & $\begin{array}{l}-0,09 * \\
(-2,56)\end{array}$ & $\begin{array}{l}-0,03^{*} \\
(-2,05)\end{array}$ \\
\hline $\begin{array}{l}\text { Austerität (Veränderung des primären Haushaltssaldos } \\
\text { ohne Zinsausgaben) }\end{array}$ & $\begin{array}{l}0,05+ \\
(1,68)\end{array}$ & $\begin{array}{l}-0,07 \\
(-0,80)\end{array}$ & $\begin{array}{c}0,03 \\
(1,08)\end{array}$ \\
\hline Wirtschaftliche Globalisierung (KOF A) & $\begin{array}{l}-0,07 \\
(-0,95)\end{array}$ & $\begin{array}{c}0,05 \\
(0,41)\end{array}$ & $\begin{array}{l}-0,17^{* *} \\
(-4,88)\end{array}$ \\
\hline Fallzahl (Regionen/Länder x Jahre) & 1117 & 538 & 579 \\
\hline Log-Likelihood & -2291 & -1110 & -1206 \\
\hline Wald_chi $^{2}$ & 42 & 113 & 263 \\
\hline$\Psi^{(3)}$ (zwischenstaatliche Varianz) & 4,64 & 4,56 & 0,88 \\
\hline$\Psi^{(2)}$ (Varianz zwischen Regionen) & 3,47 & 3,63 & 3,34 \\
\hline$\psi^{(1 *)}$ (intertemporale Varianz) & 0,47 & 0,51 & 0,21 \\
\hline$\theta$ (nicht erklärte Varianz) & 2,22 & 1,73 & 2,12 \\
\hline "Zwischenstaatliche" Intraklassenkorrelation & 0,43 & 0,44 & 0,13 \\
\hline "Interregionale" Intraklassenkorrelation & 0,32 & 0,35 & 0,51 \\
\hline Intertemporale Intraklassenkorrelation & 0,04 & 0,05 & 0,03 \\
\hline Pseudo- $R^{2}$ & 0,597 & 0,548 & 0,750 \\
\hline $\mathrm{AIC}$ & 4607 & 2244 & 2436 \\
\hline $\mathrm{BIC}$ & 4667 & 2296 & 2488 \\
\hline
\end{tabular}

$+\mathrm{P}<0,10 *{ }^{*} \mathrm{p}<0,05, * * \mathrm{p}<0,01$; in Klammern: t-Werte. Abhängige Variable: Regionale und nationale Gini-Indizes für die Ungleichheit des verfügbaren Einkommens (berechnet auf Grundlage von EU-SILC UDB 2005-2013)

Kontextvariablen von Eurostat und Dreher et al. (2008). Die abhängige Variable ist um zwei Jahre verzögert.

Quelle: Berechnungen des Autors (vgl. zum Verfahren Heidenreich 2010).

Einkommensungleichheiten überwiegend von den egalitären Strukturen und Institutionen der Nachkriegszeit maßgeblich bestimmt.

\section{Europäische und zwischenstaatliche Einkommensungleichheiten in Europa}

Die Entwicklung der Einkommensungleichheiten in Europa wird jedoch nicht nur von den bisher diskutierten innerstaatlichen Mustern und Entwicklungen bestimmt. Ungleichheiten in Europa können daher sinnvollerweise nicht mehr nur durch die Addition nationaler Ungleichheitsindizes berechnet werden. Komplementär zu einer nationalen und komparativen Perspektive ist es sinnvoll, die EU auch als einen einheitlichen sozialen Raum zu behandeln und entsprechende Ungleichheitsmaße zu berechnen.

\subsection{Innereuropäische Einkommensungleichheiten}

In Tabelle 5 werden die Ergebnisse der nach Bevölkerung gewichteten Addition der nationalen Gini-Werte und die Werte für die Einkommensungleichheit zwischen allen zur EU gehörenden Privathaushalten gegenübergestellt. Im ersten Fall wird ein Gini-Koeffizient von 30,5 für das Jahr 2013 für die EU-28 berechnet, im zweiten Fall von 33,9. Da der letztgenannte Wert auch zwischenstaatliche Ungleichheiten berücksichtigt, ist er höher als der Wert, der sich durch die Addition der (gewichteten) Ungleichheitsindizes ergibt. Er ist allerdings immer noch geringer als der vergleichbare US-amerikanische Wert (Tabelle 5) oder die 
Einkommensungleichheiten und Armut in der EU und den USA, 2013

\begin{tabular}{l|c|c|c|c}
\hline & EU-15 & $\begin{array}{c}\text { Eurozone } \\
\text { (EU-18) }\end{array}$ & EU-25 & EU-28 \\
\hline Gini (gewichtete nationale Werte) & 30,4 & 30,6 & 28,7 & 30,5 \\
\hline Gini (europaweit) & 30,0 & 30,7 & 31,8 & 33,9 \\
\hline Dezil-Verhältnis (europaweit) & 4,0 & 4,2 & 4,6 & 5,5 \\
\hline Armut (60\%; nationale Armutsschwelle) & $16,3 \%$ & $16,5 \%$ & $16,2 \%$ & $16,5 \%$ \\
\hline Armut (60\%; europäische Armutsschwelle) & $13,5 \%$ & $15,0 \%$ & $19,1 \%$ & $23,6 \%$ \\
\hline
\end{tabular}

Quelle: Berechnungen des Autors auf Grundlage von EU-SILC UDB UDB_c13_ver 2013-2 from 01-08-15; Eurostat Tabellen ilc_di12 und ilc_li02; Werte für die USA: www.lisdatacenter.org.

Werte für Bulgarien, Lettland, Litauen, Griechenland, Portugal und Rumänien (Tabelle 1). Nach einer ähnlichen Logik kann man auch Armut auf Grundlage nationaler Durchschnittseinkommen bzw. einer einheitlichen europäischen Armutsgrenze von $60 \%$ des durchschnittlichen europäischen Medians berechnen. Im ersten Fall gelten $16,5 \%$ der Europäer als arm. Legt man eine europäische Armutsschwelle zugrunde, sind 23,6\% der EU-Bevölke-

ABB. 1

\section{Entwicklung des verfügbaren Jahreseinkommens* in den verschiedenen europäischen Ländergruppen** der EU-27, 2005 - 2013 \\ Angaben in Euro \\ - - skandinavisch \\ - korporatistisch-konservativ \\ - - südeuropäisch

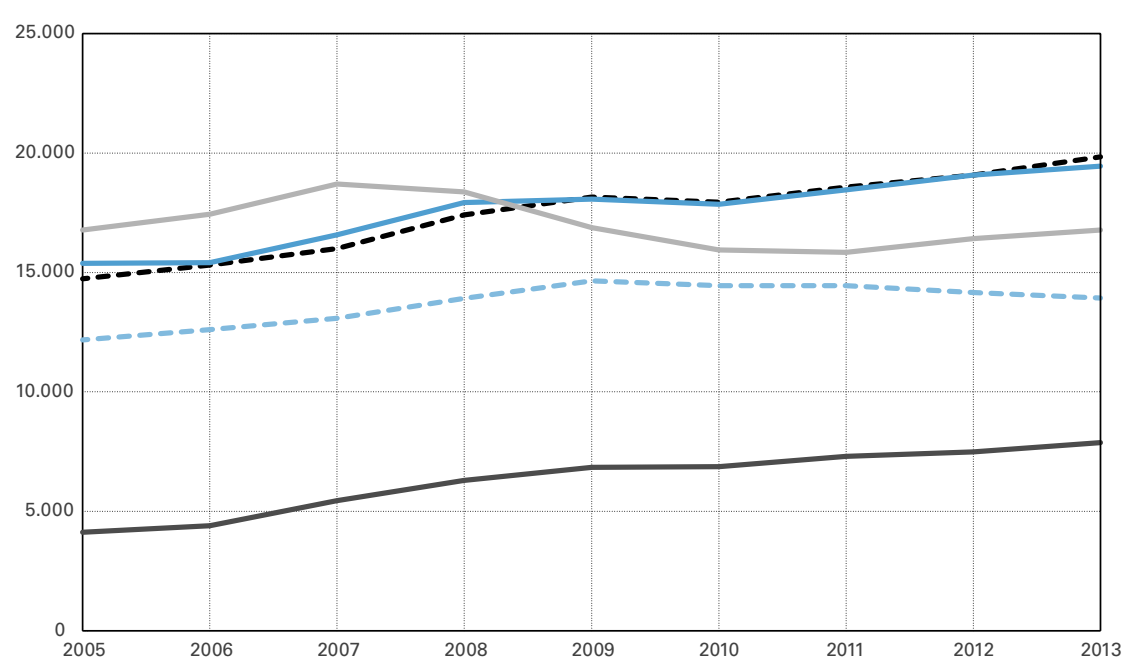

*Bevölkerungsgewichtete Durchschnitte des Haushaltsnettoäquivalenzeinkommens, in Kaufkraftstandards. **Die Länder wurden wie folgt den fünf Regionen zugeordnet: liberal: Großbritannien, Irland; korporatistisch: Niederlande Luxemburg, Frankreich, Deutschland, Belgien, Österreich; südeuropäisch: Portugal, Malta, Italien,

Spanien, Griechenland, Zypern; postsozialistisch: Rumänien, Slovakei, Polen, Lettland, Litauen,

Quelle: Eurostat, Tabelle [ilc_di04]; Berechnungen und Darstellungen des Autors. rung arm - und zwar 94,9\% der Rumänen und nur 7,6\% der Deutschen (vgl. Tabelle 3).

\subsection{Einkommensniveaus im Vergleich zwischen Wohlfahrtsregimen}

Als nächstes werden die in Tabelle 5 abgebildeten durchschnittlichen nationalen Haushaltseinkommen nach Wohlfahrtsregimen sortiert (Abbildungen 1 und 2). Die höchsten Durchschnittseinkommen weisen die skandinavischen und kontinentaleuropäischen Länder auf. Die stark finanzwirtschaftlich bestimmten angelsächsischen Länder hatten ab 2007 einen tiefen Einbruch zu verzeichnen. In den postsozialistischen Ländern Osteuropas sind die Haushaltseinkommen zwischen 2005 bis 2013 kontinuierlich angestiegen, in Südeuropa sind sie hingegen zurückgegangen. Unterhalb der gesamteuropäischen Ebene strukturiert sich somit die europäische Peripherie in der Krise um: Während die immer noch sehr großen Ost-West-Unterschiede geringer werden, nehmen die Nord-Süd-Unterschiede wieder zu. Wie Abbildung 2 zeigt, geht die Konvergenz der Einkommen mit erheblichen und zunehmenden Unterschieden bei den Arbeitslosenquoten einher. Dies wurde weiter oben als Pyrrhussieg egalitärer Institutionen bezeichnet.

\subsection{Inner- und zwischenstaatliche Einkommens- ungleichheiten im Vergleich}

Die unterschiedlichen Entwicklungen in verschiedenen Bereichen der EU lassen sich auch durch die Aufgliederung der gesamteuropäischen Einkommensungleichheit rekonstruieren (Tabelle 6). Hierbei zerlege ich die in Tabelle $2 \mathrm{ab}$ gebildete mittlere logarithmische Abweichung (MLD) in einen innerstaatlichen und einen zwischenstaatlichen Teil.

Es zeigt sich zunächst der gewaltige Anstieg der EUweiten Heterogenität durch die Osterweiterungen. Während in der EU-15 die Einkommensungleichheiten vor allem Ungleichheiten zwischen Landsleuten sind, sind die Ungleichheiten in der EU-27 noch zu fast einem Drittel Un- 
gleichheiten zwischen den Bürgern armer und reicher Länder. Seit 2007 ist eine deutliche Angleichung der Einkommensverhältnisse zu beobachten; der zwischenstaatliche Anteil der Ungleichheiten ist von 38,8 auf 30,5\% gesunken. Dies gilt auch für die Einkommensungleichheiten zwischen den EU-25-Ländern, die von 30,5\% (2005) auf nur noch 19,3\% (2013) zurückgegangen sind. Die EU ist immer noch ein zentraler Motor für die Konvergenz der Einkommensverhältnisse in Europa. Allerdings ist dieser Motor seit 2011 ins Stocken geraten.

Dem Stocken des Konvergenzprozesses liegen die schon berichteten, gegenläufigen Entwicklungen Süd- und Mittelosteuropas zugrunde (vgl. Abbildungen 1 und 2). Denn in den alten Mitgliedstaaten (EU-15) und in der Eurozone kann eine deutliche Zunahme der zwischenstaatlichen Ungleichheiten beobachtet werden (Bönke/Schröder 2015). Diese lagen im Jahr 1997 noch bei 10,7\% der gesamten Ungleichheiten der EU-15-Staaten. Die damals schon beachtliche Angleichung der Lebensbedingungen setzte sich bis zum Jahr 2010 durch den Binnenmarkt, die rechtliche Vereinheitlichung der EU, die Einführung der gemeinsamen Währung und die damit anfangs verbundenen gewaltigen Kapitalflüsse fort. 2010 betrug der zwischenstaatliche Anteil der Einkommensungleichheiten in der EU-15 nur noch 4,0\%. Im Zuge der Eurokrise jedoch kehrte sich diese Entwicklung um; der Anteil der zwischenstaatlichen Ungleichheiten ist wieder auf 9,1\% (2013) angestiegen. Ähnliches gilt für die Eurozone, in der der zwischenstaatliche Anteil von 8,7 (2010) auf 12,6\% (2013) angestiegen ist. Die Zentrum-Peripherie-Differenzen nehmen in den „alten“ Mitgliedstaaten der EU wieder zu.

Dies könnte im Sinne von Beckfield als Hinweis auf ein Ende der Angleichung gewertet werden. Angemessener erscheint es mir, sowohl die Konvergenz bis 2009 als auch die anschließende Zunahme der zwischenstaatlichen Unterschiede als Folge der Währungsunion bzw. als Ergebnis des Platzens einer „Post-Euro-Einführungsblase“ zu interpretieren. Während sich die Zinssätze nach dem Wegfall der Abwertungsrisiken 1999 bzw. mit dem Beitritt Griechenlands im Jahr 2001 insbesondere für die südeuropäischen Mitglieder der Währungsunion deutlich verringert haben, wurde 2010 klar, dass mit dem Beitritt zu einer Währungsunion, die durch eine Nichtbeistandsklausel gekennzeichnet ist, nunmehr ein Konkursrisiko verbunden war: Die Euroländer können sich nicht mehr unter Rückgriff auf eine eigene Zentralbank vor einem Staatsbankrott schützen. Weiterhin bedeutete der Verzicht auf eine eigene Währung auch den Verzicht auf das Instrument der Abwertung zur Wiedererlangung der eigenen Wettbewerbsfähigkeit, sodass gerade bei sehr niedrigen Zinsen ein Druck zur realen Senkung von Löhnen und Preisen entsteht (innere Abwertung). Allerdings haben sich die Löhne und Gehälter in den südeuropäischen Ländern als wenig anpassungsfähig erwiesen. Diese Inflexibilität bei der Lohnanpassung "nach unten“ kann als weiteres, nunmehr europäisches Sicherungsnetz für geringe Löhne verstanden werden. Allerdings wurde
ABB. 2

\section{Entwicklung der Arbeitslosenquoten* in verschiedenen europäischen Ländergruppen der EU-28, 2000 - 2014}

Angaben in Prozent der Erwerbspersonen
EU-28
- - skandinavisch
korporatistisch-konservativ
- liberal
- - südeuropäisch
- postsozialistisch

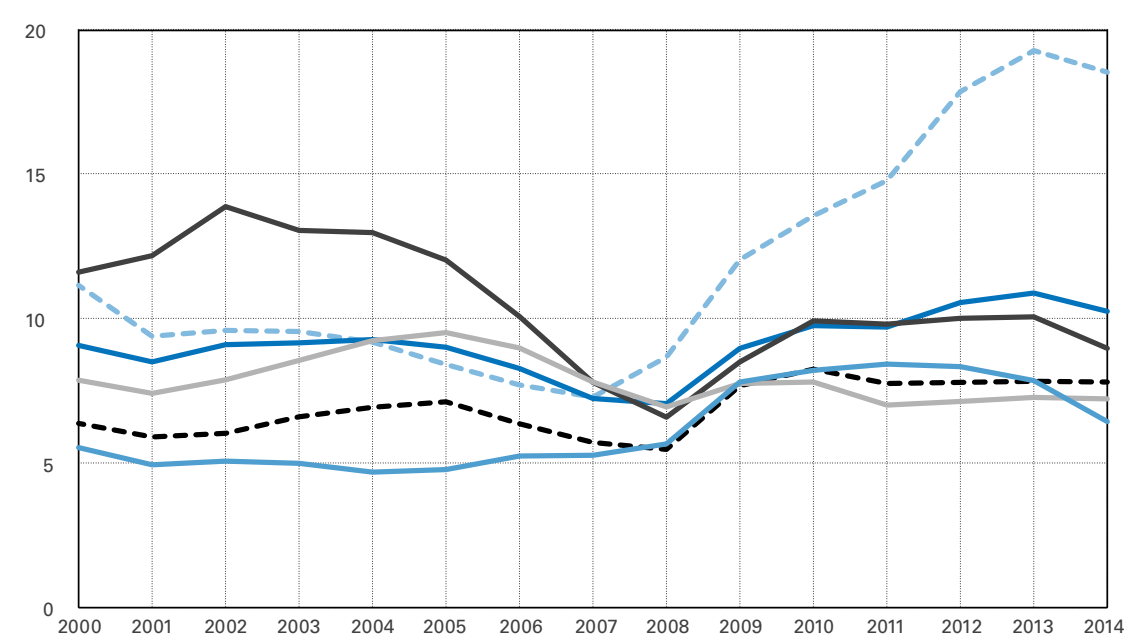

*Mit der Zahl der Erwerbspersonen gewichtete durchschnittliche Arbeitslosenquote.

Quelle: Eurostat, Tabelle [une_rt_a]; Berechnungen und Darstellung des Autors.

damit der gesamte Anpassungsdruck, der nicht mehr durch Abwertungen und nur in begrenztem Umfang durch Lohnsenkungen, Migration, Produktivitätssteigerungen, erhöhte Staatsschulden oder internationale Transfers kompensiert werden konnte, auf den Arbeitsmarkt verlagert (Streeck 2013). Dies erklärt den exorbitanten Anstieg der Arbeitslosigkeit (Abbildung 2) und die sinkenden Beschäftigungsquoten insbesondere in Südeuropa. In haushaltsbezogener Perspektive führt dies zu einer Senkung der verfügbaren Einkommen - auch für den Fall, dass die Lohnhöhe in den geschützten (und das heißt vor allem staatlichen) Bereichen der Volkswirtschaft verteidigt werden kann. Ein Gegenbeispiel wäre Irland, wo ein tiefer wirtschaftlicher Einbruch vor allem durch Lohnsenkungen bewältigt und damit der Anstieg der Arbeitslosigkeit begrenzt werden konnte. Auf diese teilweise funktionale Äquivalenz von Anpassungsmechanismen, d.h. auf den Trade-off von innerer Abwertung und Arbeitslosigkeit, sollte einleitend mit dem Bild des Pyrrhussieges aufmerksam gemacht werden.

Festgehalten werden kann, dass europaweit die Einkommensungleichheiten weitgehend stabil geblieben sind. Allerdings haben die postsozialistischen Länder Mittel- und Osteuropas im Einkommensniveau weiter aufgeholt, während die südeuropäischen Länder zurückgefallen sind. Aufgrund der institutionellen Verflechtung in einer Währungsunion ging dieser Prozess jedoch mit hoher Arbeitslosigkeit 
TABELLE 6

Entwicklung der inner- und zwischenstaatlichen Einkommensungleichheiten in der EU (mittlere logarithmische Abweichung), 1997-2013

Angaben in Prozent

\begin{tabular}{l|c|c}
\hline \multirow{2}{*}{} & \multicolumn{2}{c}{ EU 15 } \\
\cline { 2 - 3 } & Innerhalb & Zwischen \\
\hline 1997 & 89,3 & 10,7 \\
\hline 1998 & 90,3 & 9,7 \\
\hline 1999 & 92,2 & 7,8 \\
\hline 2000 & 90,6 & 9,4 \\
\hline 2001 & 90,5 & 9,5 \\
\hline $2005^{*}$ & 93,5 & 6,5 \\
\hline $2006^{*}$ & 94,3 & 5,7 \\
\hline 2007 & 93,1 & 6,9 \\
\hline 2008 & 93,5 & 6,5 \\
\hline 2009 & 95,8 & 4,2 \\
\hline 2010 & 96,0 & 4,0 \\
\hline 2011 & 95,0 & 5,0 \\
\hline 2012 & 93,0 & 7,0 \\
\hline 2013 & 90,9 & 9,1 \\
\hline
\end{tabular}

\begin{tabular}{|c|c|}
\hline \multicolumn{2}{|c}{ Eurozone (18) } \\
\hline Innerhalb & Zwischen \\
\hline & \\
\hline & \\
\hline & \\
\hline & \\
\hline & \\
\hline 86,1 & 13,9 \\
\hline 88,6 & 11,4 \\
\hline 88,6 & 11,4 \\
\hline 88,9 & 11,1 \\
\hline 91,2 & 8,8 \\
\hline 91,3 & 8,7 \\
\hline 90,6 & 9,4 \\
\hline 89,0 & 11,0 \\
\hline 87,4 & 12,6 \\
\hline
\end{tabular}

\begin{tabular}{|c|c|}
\hline \multicolumn{2}{|c}{ EU 25 } \\
\hline Innerhalb & Zwischen \\
\hline & \\
\hline & \\
\hline & \\
\hline & \\
\hline 69,5 & 30,5 \\
\hline 72,1 & 27,9 \\
\hline 72,2 & 27,8 \\
\hline 75,4 & 24,6 \\
\hline 78,5 & 21,5 \\
\hline 79,7 & 20,3 \\
\hline 81,2 & 18,8 \\
\hline 81,2 & 18,8 \\
\hline 80,7 & 19,3 \\
\hline
\end{tabular}

\begin{tabular}{|l|l}
\hline \multicolumn{2}{|c}{ EU 27 } \\
\hline Innerhalb & Zwischen \\
\hline & \\
\hline & \\
\hline & \\
\hline & \\
\hline & \\
\hline & \\
\hline & \\
\hline 61,2 & 38,8 \\
\hline 64,0 & 36,0 \\
\hline 67,1 & 32,9 \\
\hline 68,4 & 31,6 \\
\hline 69,8 & 30,2 \\
\hline 69,4 & 30,6 \\
\hline 69,5 & 30,5 \\
\hline
\end{tabular}

* Ohne Daten für Malta.

Quelle: Berechnungen des Autors auf Grundlage des ECHP und EU-SILC.

und sinkenden Beschäftigungsquoten insbesondere in Südeuropa einher. Soziale Konflikte, eine Erosion des Vertrauens in die EU und andere EU-Länder sowie das EU-weite Wachstum europaskeptischer Einstellungen und Bewegungen sind die Folge.

\section{Fazit}

Die Finanzmarkt-, Staatsschulden- und Wirtschaftskrise, die Europa seit 2008 bestimmt, hat den sozialen Zusammenhalt in der EU grundlegend erschüttert. In diesem Beitrag wurde eine mögliche Dimension dieser Erschütterungen, die Entwicklung der inner- und zwischenstaatlichen Einkommensungleichheiten, auf Grundlage europäischer Mikrodaten detailliert untersucht. Zunächst wurde eine im Durchschnitt überraschend hohe Stabilität nationaler Einkommensungleichheiten herausgearbeitet. Die bisherige Zunahme innerstaatlicher Ungleichheiten kam in der Krise zum Stillstand. Europaweit hat sich die von Beckfield in seinem Beitrag zu diesem Schwerpunktheft geäußerte Vermutung für die weitere Entwicklung bis 2013 somit nicht bestätigt. Für seine Annahme spricht eher die subjektiv verschlechterte Wahrnehmung der eigenen wirtschaftlichen Situation. Die Tatsache, dass Menschen in ärmeren Ländern ihre eigene Situation deutlich schlechter einschätzen, wird m.E. jedoch sinnvoller als Beleg für eine
Transnationalisierung bzw. Europäisierung des Bezugsrahmens interpretiert. Neben der nationalen Referenzgruppe ist auch die Position in der transnationalen bzw. europäischen Einkommenshierarchie entscheidend.

Anschließend wurde gezeigt, dass die egalitären Institutionen der Nachkriegszeit keinesfalls ihre Bedeutung verloren haben. Wohlfahrtsstaatliche Absicherungen, Gewerkschaften, qualifizierte Beschäftigte und Arbeitsverhältnisse in der Industrie gehen immer noch mit geringeren Einkommensungleichheiten einher. Dies gilt auch für eine hohe Beschäftigungsquote von Frauen, die als Hinweis auf die Wirksamkeit von Aktivierungspolitiken interpretiert wurde. Ein hohes Maß an wirtschaftlicher Integration im europäischen Binnenmarkt und mit der Welt geht in der Krise ebenfalls mit egalitäreren Einkommensstrukturen einher.

Auch europaweit zeigt sich eine hohe Stabilität der Einkommensungleichheiten. Die bisherige Abnahme zwischenstaatlicher Einkommensungleichheiten, d.h. die Konvergenz der EU-Staaten, setzte sich - im Einklang mit Beckfields These - nicht weiter fort und verkehrte sich in den EU-15-Staaten in ihr Gegenteil. Dies reflektiert vor allem die verschlechterte Einkommenssituation in den Mittelmeerländern. Während die meisten postsozialistischen Länder die Krise gut überstanden haben (vor allem die Slowakei, Polen, Rumänien und Bulgarien), gilt das nicht für die südeuropäischen Länder. Insbesondere in den fünf westeuropäischen Programmländern, die während der Krise Unterstützung durch den Internationalen Währungsfond und die europäischen Rettungsschirme 
erhalten haben (Griechenland, Zypern, Irland, Portugal und Spanien), ist eine deutliche Verschlechterung der Einkommens- und Lebensbedingungen zu beobachten. Dies ist auch als Ergebnis einer Währungsunion zu interpretieren, in der innere Abwertungen nur mit außerordentlichen Konflikten möglich sind und daher ein erheblicher Teil der Anpassungslasten auf den Arbeitsmarkt oder die Staatskasse übertragen werden. Die weitgehende Stabilität der Einkommensungleichheiten zumindest auf aggregierter Ebene ist somit ganz und gar kein Anlass zur Beruhigung. Sie reflektiert vielmehr auch die durch die Währungsunion verstärkte Europäisierung der subjektiven Ungleichheitsvorstellungen und die Stabilisierung der Reallöhne und kann nur durch die Schaffung einer entsprechenden wirtschafts- und fiskalpolitischen Kompetenz der EU institutionell abgesichert werden.

\section{LITERATUR}

Alderson, A. S./F. Nielsen (2002): Globalization and the great u-turn. Income inequality trends in 16 OECD countries, in: American Journal of Sociology 107 (5), S. $1244-1299$

Beck, U. (2012): Das deutsche Europa: neue Machtlandschaften im Zeichen der Krise. Frankfurt a. M.

Beckfield, J. (2009), Remapping inequality in Europe, in: International Journal of Comparative Sociology 50 (5-6), S. 486-504

Blyth, M. (2013): Austerity: the history of a dangerous idea, Oxford

Bönke, T./Schröder, C. (2015): European-wide inequality in times of the financial crisis, DIW-Discussion Paper (1482), Berlin

Dingeldey, I. (2011): Der aktivierende Wohlfahrtsstaat. Governance der Arbeitsmarktpolitik in Dänemark, Großbritannien und Deutschland, Frankfurt a. M.

Dreher, A./Gaston, N./Martens, P. (2008): Measuring globalisation: gauging its consequences, Wiesbaden

Europäische Kommission (2014a): Special Eurobarometer 418. Social climate, http://ec.europa.eu/public_opinion/archives/eb_special_en.htm
Europäische Kommission (2014b): Special Eurobarometer 413. Future of Europe, http://ec.europa.eu/public_opinion/archives/eb_special_en.htm Europäische Kommission (2014c): Standard Eurobarometer 81. Spring 2014 http://ec.europa.eu/public_opinion/archives/eb/eb81/eb81_vie_en.pdf Fahey, T. (2007): The case for an EU-wide measure of poverty, in: European Sociological Review 23 (1), S. 35-47

Heidenreich, M. (2003): Regional inequalities in the enlarged Europe, in: Journal of European Social Policy 13 (4), S. 313-333

Heidenreich, M. (2010): Einkommensungleichheiten in Europa, in: Zeitschrift für Soziologie 39 (6), S. 426-446

Heidenreich, M./Härpfer, M. (2010): Einkommensungleichheiten in der Europäischen Union, in: Eigmüller, M./Mau, St. (Hrsg.): Gesellschaftstheorie und Europapolitik, Wiesbaden, S. 245-273

Kenworthy, L. (2004): Egalitarian capitalism, New York

Kuznets, S. (1955): Economic growth and income inequality, in: American Economic Review 45 (1), S. 1-28

Milanovic, B. (2013): Global income inequality in numbers in history and now, in: Global Policy 4 (2), S. 198-208

OECD (Organisation of Economic Co-operation and Development) (2011): Divided we stand: why inequality keeps rising, Paris

Streeck, W. (2013): Gekaufte Zeit. Die vertagte Krise des demokatischen Kapitalismus, Frankfurt a. M.

Whelan, C. T./Maître, B. (2009): Europeanization of inequality and European reference groups, in: Journal of European Social Policy 19 (2), S. 117-130

\section{AUTOR}

MARTIN HEIDENREICH, Prof. Dr., ist Professor für Sozialstrukturanalyse an der Universität Oldenburg. Arbeitsschwerpunkte: Innovation, soziale Ungleichheiten.

martin.heidenreich@uni-oldenburg.de 